\title{
Single Shot Paravertebral Block - A Novel Approach for Post-Operative Analgesia in Breast Surgeries
}

\author{
Khushboo Jain ${ }^{1}$, Ranika Manhas², Neel Pankaj ${ }^{3}$ \\ ${ }^{1}$ Department of Anaesthesiology, Acharya Shri Chander College of Medical Sciences and Hospital, Sidhra, \\ Jammu, Jammu and Kashmir, India. ${ }^{2}$ Department of Anaesthesiology, Acharya Shri Chander College of \\ Medical Sciences and Hospital, Sidhra, Jammu, Jammu and Kashmir, India. ${ }^{3}$ Department of \\ Anaesthesiology, Bokaro General Hospital, Bokaro Steel City, Jharkhand, India.
}

\section{ABSTRACT}

\section{BACKGROUND}

Advancements in diagnostic sciences have led to increased frequency in detection of cases of breast cancer. After confirmation, majority of these patients undergo definitive surgeries commonly Modified Radical Mastectomy or Lumpectomy under general anaesthesia. In addition to inadequate pain control there is increased incidence of nausea and vomiting during first $24 \mathrm{hrs}$. of the post-operative period with GA. Parenteral narcotic used routinely in postoperative period further increases nausea and vomiting. The large number of patients hospitalized annually for breast cancer surgeries results in heavy costs and long hospital stays. Regional anaesthesia using prep-incisional paravertebral block (PVB) maybe an ideal alternative to GA alone for breast cancer surgery. Benefits include prolonged postoperative pain relief, reduction in postoperative nausea and vomiting and has potential for early discharge. It results in unilateral sensory, motor and sympathetic blockade with additional advantages of lower side effect profile, early mobilization and fewer contraindications. We wanted to study the efficacy of paravertebral block for postoperative pain relief in breast surgeries.

\section{METHODS}

A prospective, randomized, comparative study involving 60 adult female patients posted for Ca breast surgery was conducted. One group received pre-incisional PVB with GA (group A) and another received GA alone (group B). The efficacy of analgesia and PONV were assessed using Visual Analogue Scale and Numeric Rating Score respectively at T1, 2, 3, 4, 5, 6, 12, 24, 48 hours. Fentanyl 2 mic / Kg as rescue analgesic and ondansetron $0.1 \mathrm{mg} / \mathrm{Kg}$ as antiemetic were given at VAS $>/=4$ and NRS $>/=2$. Total opioid and antiemetic consumption was noted.

\section{RESULTS}

Total VAS and NRS scores of Group A was significantly lower than Group B. Also significantly reduced consumption of analgesic and antiemetic was observed in Group A. $\sum \mathrm{VAS}_{\mathrm{A}}=(3.37+2.76)$ while $\sum \mathrm{VAS}_{\mathrm{B}}=(19.23+3.32)$ while, Group A $\sum \mathrm{NRS}$ $=(0.47+0.67)$ and Group B $\sum \mathrm{NRS}=(5.27+1.34)$.

\section{CONCLUSIONS}

PVB provides significant pain relief with decreased incidence of PONV and has the additional advantage of lesser consumption of opioids and antiemetics in the immediate postoperative period.

\section{KEY WORDS}

Pre-incisional, Paravertebral Block, Breast Surgery, Single Shot Approach, Postoperative Analgesia
Corresponding Author:

Ranika Manhas,

Acharya Shri Chander College of Medical

Sciences and Hospital, Sidhra, Jammu,

Jammu and Kashmir, India.

E-mail:ranikamanhas@gmail.com

DOI: $10.14260 /$ jemds/2020/609

How to Cite This Article:

Jain K, Manhas R, Pankaj, Pankaj N. Single shot paravertebral block- a novel approach for postoperative analgesia in breast surgeries. J Evolution Med Dent Sci 2020;9(38):2796-2800, DOI: $10.14260 / \mathrm{jemds} / 2020 / 609$

Submission 12-03-2020,

Peer Review 25-04-2020,

Acceptance 04-05-2020,

Published 21-09-2020.

Copyright (C) 2020 JEMDS. This is an open access article distributed under Creative Commons Attribution License [Attribution 4.0 International (CC BY 4.0)] 


\section{BACKGROUND}

Advancements in diagnostic sciences have led to increased frequency in detection of cases of breast cancer. After confirmation, majority of these patients undergo definitive surgeries commonly Modified Radical Mastectomy or Lumpectomy under general anaesthesia. General anaesthesia (GA) is currently the standard technique used for surgical treatment of breast cancer. However, the side effects and complications of GA preclude ambulatory surgery for most patients undergoing breast surgery. In addition to inadequate pain control, a 59\% incidence of nausea and vomiting during first $24 \mathrm{hrs}$ postoperative with GA has been reported. ${ }^{1}$ Parenteral narcotic used routinely in postoperative period further increases nausea, vomiting and sedation. The large number of patients hospitalized annually for Breast cancer surgeries entails heavy cost. Recent efforts are focused at reducing hospital cost and stay. ${ }^{2}$

Regional anaesthesia using pre-incisional paravertebral block (PVB) maybe an ideal alternative to GA alone for breast cancer surgery. Benefits include prolonged postoperative pain relief, reduction in postoperative nausea and vomiting and potential for early discharge. ${ }^{3}$ Chronic pain symptoms in the operated area and the ipsilateral arm are prevalent even one year after breast surgery. Good immediate postoperative analgesia is achieved by providing pre-incisional PVB in patients undergoing breast surgery for cancer. Good acute pain relief is associated with lower risk of development of chronic pain at operated area

Thoracic paravertebral block involves injection of local anaesthetic in paravertebral space which contains dorsal and ventral rami and sympathetic chain. Infiltration in this space results in unilateral sensory, motor and sympathetic blockade. Para vertebral block has also been used to relieve acute chest wall pain from rib fracture, herpes zoster, pleurisy, to manage acute and chronic post thoracotomy pain and as an anaesthetic technique for chest wall surgeries.

In comparing analgesia obtained from epidural versus paravertebral, side effects of postural hypotension, urinary retention are significant problems with epidural analgesia. In comparing intercostal block with paravertebral block, intercostal block has inherent limitations of inadequate spread at multiple levels, with an additional inadequate analgesia and greater rates of complications of pleural or pulmonary damage. Intrapleural analgesia leads to significantly worsened pulmonary function in comparison to paravertebral block. Dependent chest drains losses of local anaesthetics are much less with paravertebral blocks. Risk of pleural and pulmonary damage with intrapleural blocks is greater than with paravertebral technique.

Hence, PVB provide excellent pain relief and inhibit the neuro endocrine stress response to surgical trauma which suggests that a very high-quality afferent block can be effective. PVB is relatively easy to learn, have fewer contraindications and require no additional nursing surveillance. They are applicable to large number of patients and because of their low side effect profile they contribute to early postoperative mobilization and reduced hospital stay. In this study our primary hypothesis was that paravertebral block using $0.25 \%$ bupivacaine would have better postoperative pain control along with lower incidence of postoperative nausea and vomiting and opioid and antiemetic consumption as compared to GA alone.

\section{METHODS}

After the approval of the institutional ethical committee, this randomized controlled study was conducted among 60 adult female patients. Sample size was calculated by Sample Size Calculator (Creative Research System Survey software) using the following assumptions and it was found to be 60 with a confidence level $=95 \%$, Confidence interval $=11.33$ and $\mathrm{a}$ Population size of 300 . The patients posted for mastectomy surgery under general anaesthesia during study period are referred to as population group. A rough estimate of the strength of this group was made by forward regression of previous year data of annual mastectomy surgeries under general anaesthesia. Written informed consent was obtained and these patients were randomly allocated into two groups of 30 each. (randomization done by a random number table generated online (https://www.random.org/intergers)

Inclusion criteria were Adult female patients who were diagnosed case of Breast Cancer, aged between 20 - 60 years, ASA I and ASA II physical status, scheduled for elective breast surgery.

Exclusion criteria were Patients refusal and un cooperative patients, Any patient other than ASA I or ASA II, Severe obesity (BMI $\left.>35 \mathrm{~kg} / \mathrm{m}^{2}\right)$,Pregnancy or breast feeding females, Infection at the site of injection, Bleeding disorders, Allergy to amide type local anaesthetic, Patients with past history of musculoskeletal disorders, Additional surgical procedure during -the same surgical time.

On the day of surgery an intravenous access was obtained using 18G intravenous cannula. Premedication was provided $45 \mathrm{~min}$ before induction with glycopyrrolate ( $4 \mathrm{mcg} / \mathrm{Kg}$ ) and fentanyl ( $2 \mathrm{mcg} / \mathrm{Kg}$ ) IV. Paravertebral block was performed in patients of group A by the attending anaesthesiologist. Needle Insertion Point was $2.5 \mathrm{~cm}$ lateral to the tip of spinous process at the level of T3 on the side of surgery. Part was cleaned and painted with antiseptic solution. Sterile drape was placed. Planned needle insertion point was infiltrated with $5 \mathrm{~mL}$ of $2 \%$ lignocaine. A $16 \mathrm{G}$ needle was inserted perpendicular to the skin to contact transverse process at 2-4 $\mathrm{cm}$ depth. Then the needle was manipulated to walk off the superior aspect of transverse process until loss of resistance was felt. Insertion was limited to $<2 \mathrm{~cm}$ past the transverse process. $20 \mathrm{~mL}$ of $0.25 \%$ bupivacaine was injected. Dose used was 3 - $4 \mathrm{~mL} /$ dermatome. Patient was made to lie down supine. Onset of sensory anaesthesia was checked 10-15 min after the injection by needle prick technique. If the patient had lack of sensory blockade in 10 - 15 min it was considered as failed Paravertebral Block and the patient was excluded from the study.

GA was induced in both the study groups with Fentanyl 1 mic / Kg IV, Propofol 2 mg / Kg IV, Succinylcholine 1.5 mg / $\mathrm{Kg}$ IV was given to facilitate intubation. After intubation GA was maintained with Sevoflurane $0.6 \%$ with $66 \%$ nitrous oxide in $33 \%$ oxygen. Neuromuscular blockade was achieved using Vecuronium $0.1 \mathrm{mg} / \mathrm{Kg}$ IV. Heart rate, noninvasive blood pressure, $\mathrm{SpO} 2$, end tidal $\mathrm{CO}_{2}\left(\mathrm{EtCO}_{2}\right)$ and ECG was 
monitored. Ondansetron 0.15 mcg / Kg IV was given 30 min prior extubation. Residual neuromuscular blockade was reversed with IV Neostigmine $50 \mathrm{mcg} / \mathrm{Kg}$ and Glycopyrrolate $8 \mathrm{mcg} / \mathrm{Kg}$. After surgery patients were observed in post op room for 2 hours and then were shifted to respective wards. for grading of pain at operation site or radiating to arm or axilla. VAS scores were recorded on 1st hour(T1), 2nd hour (T2), 3rd hours(T3), 4th hour (T4), 5th hour (T5), 6th hour (T6), 12th hour (T12), 24th hour (T24) and 48th hour (T48) postoperatively. Also, postoperative nausea and vomiting was assessed using NUMERIC RATING SCALE (NRS).

In both the groups rescue analgesia was given with Fentanyl $2 \mathrm{mcg} / \mathrm{Kg}$ to patients with VAS scores of $>/=4$. Ondansetron $0.1 \mathrm{mg} / \mathrm{Kg}$ was given as antiemetic to patients with NRS $>/=2$. Total required doses of both the drugs were recorded for entire duration of observation.

Patients were monitored intra operatively immediately after giving PVB and post operatively Patient in group A were observed for complications, viz: -Pneumothorax, Hypotension, Dural puncture related complications e.g. PDPH, intrathecal injection, pulmonary haemorrhage, Hematoma, Local anaesthetic toxicity.

\section{Statistical Analysis}

The observations recorded in each group were compared using statistical analysis. After the collection of data mean and standard deviation were calculated for each variable in both the groups. The apparent difference in mean values of both the groups were compared using standard error (SE) of difference between means and standard error (SE) of difference between proportions. If the two means of two groups were found to be separated by $>2$ SE then two means will be considered as significant $(\mathrm{p}<0.05)$ apart.

Student's Paired t test was used since it was normally distributed data. Software used for calculation of $p$ value was Stata (version 10).

\section{RESULTS}

Demographic data of the two groups Group A (GA + PVB) and Group B (GA) were comparable in terms of age, weight, height and BMI. There was no statistically significant difference in two groups. (table 1).

VAS and NRS scores of both the groups were measured. VAS scores of Group A was found to be significantly lower than Group B for first postoperative day. $\sum \mathrm{VAS}_{\mathrm{A}}=(3.37+$ 2.76) while $\sum \operatorname{VAS}_{B}=(19.23+3.32)$. NRS scores of Group $A$ was found to be significantly lower than Group B Group A $\sum N R S=(0.47+0.67)$ while Group B $\sum N R S=(5.27+1.34)$ as shown in Figure $1 \& 2$. Opioid consumption in Group B was found to be significantly greater than group A. Similarly, antiemetic consumption in Group B was found to be significantly greater than group A as shown in Figure $3 \& 4$.

Not any of the above-mentioned complications were noted in any patient.
Patients were instructed on the use of VAS scale $(0-10)$

\begin{tabular}{|c|c|c|c|}
\hline Demographic Variable & Group A & Group B & p Value \\
\hline Age (Years) & $47.33 \pm 8.33$ & $47.06 \pm 9.20$ & 0.45 \\
\hline Weight (Kilograms) & $59.63 \pm 7.95$ & $58.76 \pm 13.81$ & 0.33 \\
\hline Height (cm) & $157.9 \pm 7.10$ & $159.3 \pm 7.33$ & 0.23 \\
\hline BMI $\left(\mathrm{Kg} / \mathrm{m}^{2}\right)$ & $24.01 \pm 3.63$ & $23.88 \pm 3.30$ & 0.63 \\
\hline \multicolumn{3}{|c|}{ Table 1. Demographic Data } \\
\hline
\end{tabular}
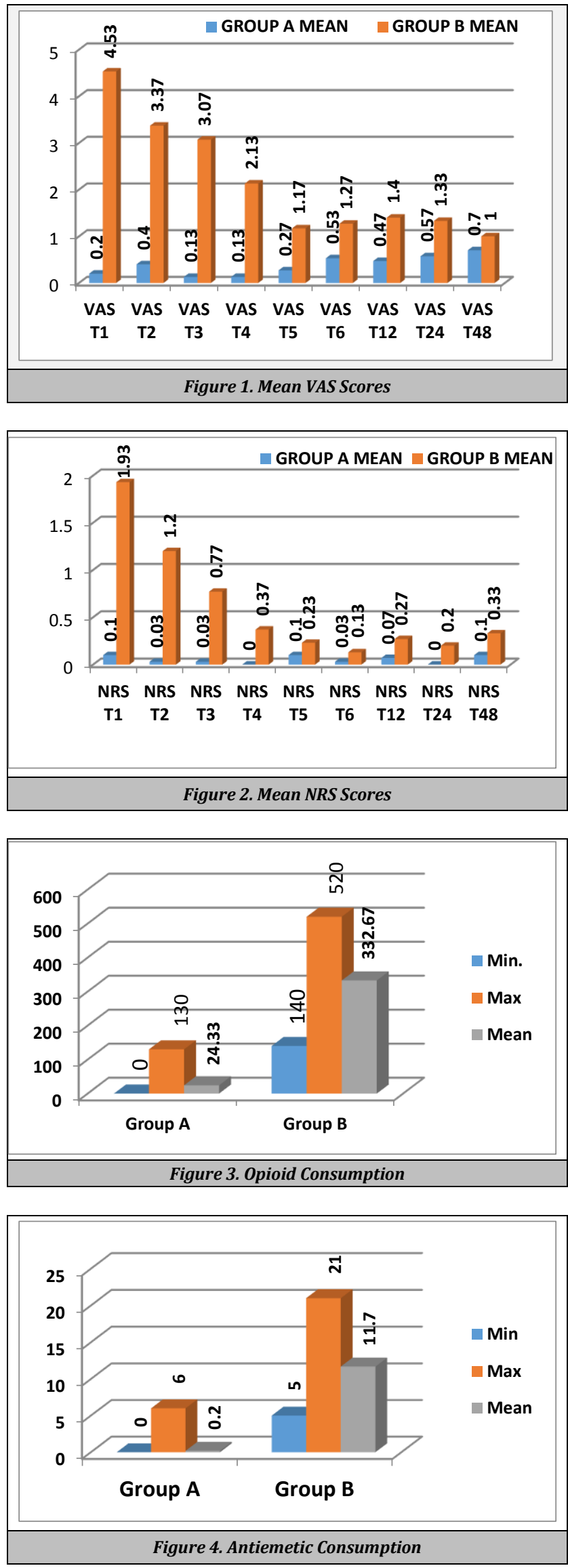


\section{DISCUSSION}

In the recovery room, most of the post-operative patients require opioids and antiemetics for pain and nausea vomiting. Opioid use further increases the incidence of postoperative nausea vomiting. These side effects preclude ambulatory surgery for most of the patients. Chronic pain symptoms in the operated area and the ipsilateral arm are prevalent even one year after breast surgery 5 Good acute pain relief causes lesser incidence of postoperative chronic pain. Alternative approaches need to be employed for better immediate recovery and further reducing chronic pain.

Paravertebral Block using $0.25 \%$ bupivacaine can be an ideal alternative or adjunct to General anaesthesia for breast cancer surgery. It acts by direct penetration of local anaesthetic into the dorsal ramus, rami communicantes and intercostal nerve. A thoracic paravertebral injection of local anaesthetics results in ipsilateral somatic and sympathetic nerve block including the posterior ramus in multiple contiguous thoracic dermatomes. Hence, blockade at single level results in multi dermatomal spread of the drug. Also, pre incisional PVB inhibit the neuro endocrine stress response to surgical trauma which suggests that a very high quality afferent block can be effective. ${ }^{6}$ Total blockade would remove the stimulus for central sensitization and hence the augmentation of nociception, which is thought to be responsible for postoperative and chronic pain. ${ }^{7}$ The spinal nerves in this space are devoid of a fascial sheath, making them exceptionally susceptible to local anaesthetics.

In our study the patients receiving pre incisional Paravertebral block with $20 \mathrm{~mL}$ of $0.25 \%$ bupivacaine i.e., Group A, had statistically significant pain relief for first 24 hours post operatively $(\mathrm{p}<0.05)$ than Group B receiving GA only. Overall $\sum \mathrm{VAS}_{\mathrm{A}}$ was $3.37 \pm 2.76$ while $\sum \mathrm{VAS}_{\mathrm{B}}$ was 19.23 \pm 3.32 .

Similar results of significantly reduced postoperative pain with thoracic paravertebral block in elective Ca breast surgeries has been observed by Kairaluoma et al8in 2004 and Dabbagh et $\mathrm{al}^{9}$ in 2007 suggesting that the pre incisional PVB inhibits neuro endocrine stress response to surgical trauma which suggests that a very high quality afferent block can be effective $^{6}$ in attenuation of pain. Also, Kairaluoma et $\mathrm{al}^{10}$ observed lesser prevalence of chronic pain in the preincisional PVB group of patients in 2006 thus reinforcing that significantly low VAS score attributed to total blockade of somatic, motor and sympathetic fibres would remove the stimulus for central sensitization and hence the augmentation of nociception, which is thought to be responsible for postoperative and chronic pain. ${ }^{7}$ The significantly longer duration of postoperative analgesia with thoracic paravertebral block as observed by Greengrass et al ${ }^{11}$ for an average of 23 hours (range 9-38 hours) and Klein et al ${ }^{12}$ and Naja et $\mathrm{al}^{13}$ for up to 72 hrs supports the fact that spinal nerves in this space are devoid of a fascial sheath, making them exceptionally susceptible to local anaesthetics. Apart from breast surgeries, efficacy of PVB was also proved in thoracoscopic surgeries deriving similar conclusions in 2005 by Vogt et al. ${ }^{14}$

Regarding secondary outcome of Postoperative Nausea and Vomiting (PONV), total Antiemetic and Analgesic consumption in postoperative period, incidence of PONV is higher after general anaesthesia, in female sex and aggravated by postoperative pain. Parenteral narcotic use further increases incidence of PONV and recovery room stay. NRS scores of both groups were compared, Group A $\sum$ NRS = $(0.47 \pm 0.67)$ had significantly lower scores than Group B $\sum \mathrm{NRS}=(5.27 \pm 1.34) ; \mathrm{p}<0.05$. Group B significantly has much more consumption of analgesic and antiemetic post operatively than Group A ( $\mathrm{p}<0.0001$ for both opioid and antiemetic consumption).

Hence, apart from the confounding factors higher use of postoperative narcotics in only GA group, as compared to pre-incisional PVB with GA group, resulted in higher incidence of Postoperative nausea and vomiting as observed by Kairaluoma et al. ${ }^{8}$ Also, in studies where GA was compared with sole PVB similar conclusions of much reduced incidence of postoperative nausea vomiting and opioid and antiemetic consumption was noted with PVB group by Naja et al ${ }^{13}$ in 2003, Youssef Tahiri et el ${ }^{15}$ in 2011 and Fahy et al ${ }^{16}$ in 2014. The lower nausea and vomiting scores in the PVB group is attributed to improved analgesia and the need for less postoperative opioids. The use of paravertebral nerve blockade was also associated with a significantly shorter hospital stay (median 1 day) compared to general anaesthesia ( 2 days) $(\mathrm{p}<0.01)^{13}$

In our study none of the above-mentioned complications were observed regarding the technique of Paravertebral Block both intraoperatively and post operatively. An epidural extension in 2 out of 156 patients were observed by Coveney et $\mathrm{l}^{17}$ while accidental intravascular injection was reported in one of the cases by Kairaluoma et $\mathrm{al}^{8}$. Terheggen et $\mathrm{al}^{18}$ reported one inadvertent epidural block and one inadvertent pleural puncture in his study. Nil complications were reported by Greengrass et al, ${ }^{11}$ Klein et al ${ }^{12}$ and Moller et al. ${ }^{19}$

\section{Limitations}

Blinded studies are more accurate, double blinding using sham block with saline can be implemented in further studies. Stress testing by measurement of blood cortisol levels and other mediators could be instituted for more precise grading of pain. Ultrasound guided PVB would further reduce the complication rate and would increase the efficacy and precision of the block and addition of adjuvants or using different local anaesthetics might further increase duration of pain relief or reduced incidence of PONV.

\section{CONCLUSIONS}

Paravertebral block when used in conjunction with general anaesthesia provides superior analgesia in the postoperative period in comparison to general anaesthesia alone for the first 24 hours $(\mathrm{p}<0.05)$, further reducing the incidence of postoperative nausea and vomiting in comparison to general anaesthesia alone. NRS scores were significantly low in Group A than Group B

Paravertebral block leads to significantly reduced consumption of opioids and antiemetics in the postoperative period in comparison to general anaesthesia alone. Complication rates of paravertebral block are significantly 
low thereby proving it to be a relatively safe procedure. The ability to avoid hospitalization and recover at home without pain, nausea, and vomiting softens the impact of a cancer diagnosis and encourages early return to normal activity or initiation of further treatment"

Financial or Other Competing Interests: None.

\section{REFERENCES}

[1] Miguel R, Rothschiller J, Majchrzak J. Breast surgery is a high risk procedure for development of nausea and vomiting. Anaesthesiology 1993;79:A1095.

[2] Cohen AM, Schaeffer N, Chen ZY, et al. Early discharge after modified radical mastectomy. Am J Surg 1986;151(4):465-6.

[3] Richardson J, Sabanathan S. Thoracic paravertebral analgesia. Acta Anaesthesiol Scand 1995;39(8):1005-15.

[4] Perkins FM, Kehlet H. Chronic pain as an outcome of surgery: a review of predictive factors. Anaesthesiology 2000;93(4):1123-33.

[5] Tasmuth T, von Smitten K, Kalso E. Pain and other symptoms during the first year after radical and conservative surgery for breast cancer. Br J Cancer 1996;74(12):2024-31.

[6] Richardson J, Sabanathan S, Mearns AJ, et al. Efficacy of pre-emptive analgesia and continuous extrapleural intercostal nerve block on post-thoracotomy pain and pulmonary mechanics. J Cardiovasc Surg (Torino) 1994;35(3):219-28.

[7] Wall PD. The prevention of postoperative-pain. Pain 1988;33(3):289-90.

[8] Kairaluoma PM, Bachmann MS, Korpinen AK, et al. Single injection paravertebral block before general anaesthesia enhances analgesia after breast cancer surgery with and without associated lymph node biopsy. Anaesth Analg 2004;99(6):1837-43.

[9] Dabbagh A, Elyasi H. The role of paravertebral block in decreasing postoperative pain in elective breast surgeries. Med Sci Monit 2007;13(10):CR464-7.
[10] Kairaluoma PM, Bachmann MS, Rosenberg PH, et al. Preincisional paravertebral block reduces the prevalence of chronic pain after breast surgery. Anesth Analg 2006;103(3):703-8.

[11] Greengrass R, O’Brien F, Lyerly K, et al. Paravertebral block for breast cancer surgery. Can J Anaesth 1996;43(8):858-61.

[12] Klein SM, Bergh A, Steele SM, et al. Thoracic paravertebral block for breast surgery. Anesth Analg 2000;90(6):1402-5.

[13] Naja MZ, Ziade MF, Lönnqvist PA. Nerve-stimulator guided paravertebral blockade vs. general anaesthesia for breast surgery: a prospective randomized trial. European Journal of Anaesthesiology 2003;20(11):897903.

[14] Vogt A, Stieger DS, Theurillat C, et al. Single injection thoracic paravertebral block for postoperative pain treatment after thoracoscopic surgery. Br J Anaesth 2005;95(6):816-21.

[15] Tahiri Y, Tran DQH, Bouteaud J, et al. General anaesthesia versus thoracic paravertebral block for breast surgery: a meta-analysis. J Plast Reconstr Aesthet Surg 2011;64(10):1261-9.

[16] Fahy AS, Jakub JW, Dy BM, et al. Paravertebral blocks in patients undergoing mastectomy with or without immediate reconstruction provides improved pain control and decreased postoperative nausea and vomiting. Ann Surg Oncol 2014;21(10):3284-9.

[17] Coveney E, Weltz CR, Greengrass AG, et al. Use of paravertebral block anaesthesia in the surgical management of breast cancer: experience in 156 cases. Annals of Surgery 1998;227(4):496-501.

[18] Terheggen MA, Wille F, Rinkes IHB, et al. Paravertebral blockade for minor breast surgery. Anesth Analg 2002;94(2):355-9.

[19] Moller JF, Nikolajsen L, Rodt SA, et al. Thoracic paravertebral block for breast cancer surgery: a randomized double blind study. Anesth Analg 2007;105(6):1848-51. 